\title{
An Intelligent Trading System with Fuzzy Rules and Fuzzy Capital Management
}

\author{
Rodrigo Naranjo, ${ }^{1}$ Albert Meco, ${ }^{2}$ Javier Arroyo, ${ }^{3}$ Matilde Santos ${ }^{3,}$ \\ ${ }^{1}$ Instalaciones Inabensa, Abengoa, Seville, Spain \\ ${ }^{2}$ Materia Works S.L., Madrid, Spain \\ ${ }^{3}$ Facultad de Informática, Universidad Complutense de Madrid, 28040-Madrid, Spain
}

*Author to whom all correspondence should be addressed; e-mail: msantos@ucm.es.

In this work we are proposing a trading system where fuzzy logic is applied not only for defining the trading rules, but also for managing the capital to invest. In fact, two fuzzy decision support systems are developed. The first one uses fuzzy logic to design the trading rules and to apply the stock market technical indicators. The second one enhances this fuzzy trading system adding a fuzzy strategy to manage the capital to trade. Additionally, a new technical market indicator that produces short and long entry signals is introduced. It is based on the MACD (Moving Average Convergence Divergence) indicator. Its parameters have been optimized by genetic algorithms. The proposals are compared to a classical non-fuzzy version of the proposed trading systems and to the Buy-and-Hold strategy. Results favor our fuzzy trading system in the two markets considered, NASDAQ100 and EUROSTOXX. Conclusions suggest that the use of fuzzy logic for capital management is promising and deserves further exploration. (c) 2015 Wiley Periodicals, Inc.

Keywords: trading, fuzzy logic, decision making, optimal-F, capital management

\section{INTRODUCTION}

In finance, the Efficient Market Hypothesis (EMH) states that financial markets are "informationally efficient". That is, existing share prices always incorporate and reflect all relevant information. In consequence, it is impossible for investors to either purchase undervalued stocks or sell stocks for inflated prices. The only way an investor can possibly obtain higher returns is by purchasing riskier investments. In other words, the time series of the returns from speculative assets are "unforecastable". Some authors have studied in depth the implications of the EMH and offer guidance about the kind of forecasting approaches that may work even in financial markets. ${ }^{1}$ However, they acknowledge that new financial prediction methods are expected to offer only short-lived profits, because once these methods become more widely used, their 
information may get incorporated into prices and they will cease to be successful. This frustrating perspective has not discouraged researchers and practitioners from seeking for a "money machine", even if it is expected to have a short life span.

In the literature mixed evidence on the profitability of the technical trading rules can be found. In Ref. 2 and 3 showed that technical trading rules are profitable. Gençay ${ }^{4}$ also approved that a more sophisticated approach that combines nonparametric models with technical strategies provided significant profits when tested against Buy \& Hold strategies. More recently, Vasileiou ${ }^{5}$ proved how even the simplest trading rules could take advantage of the market's inefficiency and lead to profitable trading decisions. On the other hand, it seems that trading rules are not so profitable since 1986 due to their widespread use once their initial success. ${ }^{6}$ Bajgrowicz and Scaillet ${ }^{7}$ revisited the apparent historical success of technical trading rules on daily prices of the Dow Jones Industrial Average index from 1897 to 2011. They showed that an investor would never have been able to select the future best-performing rules and concluded that their results seriously call into question the economic value of technical trading rules that has been reported for early periods. As it can be seen, the debate on the profitability of the simplest strategies is still open.

The rise of soft computing methods in the last few decades have also led to the exploration of more sophisticated approaches for the prediction of stock markets. As a result, intelligent proposals such as optimization by genetic algorithms, ${ }^{8,9}$ nonlinear models based on neural networks, ${ }^{10,11}$ fuzzy logic, ${ }^{12,13}$ etc., have become popular tools amongst quantitative traders. Atsalakis and Valavanis ${ }^{14}$ surveyed more than 100 related published articles that focus on neural and neuro-fuzzy techniques derived and applied to forecast stock markets. Through the surveyed papers, they showed that soft computing techniques are widely accepted to studying and evaluating stock market behavior. Similarly Krollner et al. ${ }^{15}$ presented a survey of the recent literature on machine learning techniques and artificial intelligence used to forecast stock market movements.

Some approaches combine different Soft Computing methodologies, leading to even more sophisticated hybrid methods that aim to predict the markets. For example, an intelligent decision-making model to generate one-step forward investment decisions for stock markets was developed by the application of fuzzy neural networks and swarm intelligence. ${ }^{16}$ Particle Swarm Optimization algorithm was applied to select the best neural network for the future investment decisions. In a similar way Bagheri et al. ${ }^{17}$ presented a hybrid intelligent method to forecast financial time series. Their methodology combined wavelet transform for the full decomposition of the time series as inputs of an Adaptive Network-based Fuzzy Inference 
System and at the same time, quantum-behaved Particle Swarm Optimization to tuning the ANFIS membership functions. This combination of soft computing techniques was also shown in Ref. 18 by an integrated approach based on genetic fuzzy systems and artificial neural networks (particularly, self-organizing map network) for the construction of an expert system that predicts market prices. A further example that combines rough sets theory and genetic algorithms for stock price forecasting is shown in Ref. 19. Lam et al. ${ }^{20}$ proposed a stock market timing system integrating a genetic algorithm with a fuzzy expert system. Genetic algorithm is used to optimize the selection of the fuzzy trading rules and the weights of these rules. In this line, Kuo et al. ${ }^{21}$ incorporated not only the technical indexes (quantitative factors) but also qualitative factors (i.e., knowledge of stock market experts) in a fuzzy neural network based on genetic algorithms to develop the knowledge base of the fuzzy inference rules that can measure the qualitative effect on the market. The references in this article are only a small sample of the literature on predicting stock markets, using Soft Computing methods.

However, forecasting the market behavior (including value, direction, volatility or signals for enter and exit trades) is only one aspect of a trading system. Trading systems are mainly decision support systems that transform forecasts into buy/sell orders to exploit the "pockets of predictability" in financial markets. These decision support systems, according to Chande, ${ }^{22}$ consist of three main functions, namely: i) Rules to enter and exit trades; ii) Risk control, and iii) Money management. It is surprising to find far fewer applications of soft computing methods dedicated to ii) and iii). Risk control is the strategy that can be used to protect the trading operations. The simplest strategies are Stop-Loss and Take-Profit orders, which are usually adjusted according to the market's volatility. Another important aspect of trading systems is that they should manage capital in an efficient way. Different strategies are used by technical traders to determine the appropriate amount of money to invest each time: the Kelly criterion, ${ }^{23,24}$ the optimal- $\mathrm{F}^{25}$ and the fixed-ratio. ${ }^{26}$

This paper attempts to show how a given technical trading system can be enhanced with the help of fuzzy logic on both aspects, the definition of the trading rules and the money management. Fuzzy logic trading systems are easy to comprehend and modify, what benefits them against hybrid approaches that are often too cumbersome for traders.

The use of fuzzy logic in trading rules has been successfully explored in some works, to represent the inherent uncertainty of trading signals and to incorporate the expertise of the trader in the system. However, its use in money management is a less beaten track. But in capital management, fuzzy logic enables a more 
conservative decision than recommendations to buy or sell which invest all available funds in one position. On this point, our work is similar to the one by Gradojevic and Gençay, ${ }^{27}$ in the sense that our fuzzy trading system addresses the dual purpose of market timing ("when to trade") and order size (" how much to trade'’).

Regarding the trading rules, they can be based on charting and/or on technical indicators. Charting is mainly a visual activity, looking for patterns in the price time series. There are examples of fuzzy logicassisted charting such as the work by Zhou and Dong, ${ }^{13}$ who used a fuzzy representation of the time series in order to look for some of the well-known pattern templates in technical trading, and Lee et al. ${ }^{12}$ who proposed a fuzzy representation of candlestick time series data. On the other hand, technical indicators are used to forecast the direction of prices, to identify good entry and exit points for the trade, or to determine the long-term trend. There is a diverse catalogue of indicators, ${ }^{28}$ and also some technical traders create their own indicators to guide them in the decision making. Other works proposed fuzzy versions of technical financial indicators to control the uncertainty that arises due to the potential imprecision, incompleteness and unreliability of the technical indicators themselves. For instance, Dourra and Siy ${ }^{29}$ proposed the fuzzification of technical indicators and rules to generate buy, sell and hold signals. Gradojevic and Gençay ${ }^{27}$ combined standard moving average and filter strategies with fuzzy control methodology, which results in improved profitability. Escobar et al. ${ }^{30}$ created their own fuzzy indicator that uses as inputs the risk aversion of the investor and market information such as the profitability and volatility of the stock prices, while the outputs are the buy and sell signals.

We present herein an adaptation of a market strategy to the fuzzy context and investigate the role of fuzzy logic in a trading system. Both the trading rules and the technical indicators are based on the fuzzy approach. The output of the decision system is also a fuzzy variable that represents the uncertainty of the prediction that characterizes the buy-sell signals. The proposed intelligent decision system provides a conservative risk management strategy using stop-loss and stop-profit orders, but it also includes a fuzzy estimation of the amount to be invested based on the optimal-F formula. ${ }^{25}$

Finally, our fuzzy trading system has been compared to two versions of the same trading system, one semi-fuzzy and one non-fuzzy, and to the Buy \& Hold strategy, which is a standard benchmark in the literature, see for example..$^{19,20,27}$ The results obtained are promising in terms of profitability. It also shows how to apply fuzzy logic in trading systems in a simple but yet effective way. 
The remainder of this paper is organized as follows: In section 2 the trading system is explained and the fuzzy indicators are detailed, including the new proposed in this paper. Section 3 focuses on classical and fuzzy money management techniques implemented in our decision system. Section 4 presents the experimental set up and compares and discusses the results obtained by the fuzzy and analytic methods. Section 5 concludes the paper and suggests some possible future work.

\section{TRADING SYSTEM}

In this section, the main components of the intelligent trading system developed are described. First we consider a system based on a classical strategy, as it would be used by a technical trader. Then we proposed two fuzzy versions of the trading process; on of the rules and the other one which includes capital management. The trading system is based on the use of two known technical indicators and a third one proposed by us.

\subsection{Technical Indicators}

The field of technical analysis dates back to the early twentieth century when Charles Dow wrote a series of articles describing systematic phenomena in the stock markets. Since then many types of indicators have been proposed, see for instance. ${ }^{28,31}$ The most popular ones are the moving averages, but also more sophisticated indicators are widely used by technical traders.

In our case we used a set of three technical indicators. Two of them are well known RSI (Relative Strength Index) and ADX (Average Directional Movement Index); the first is a momentum oscillator that accounts the increase or fall in price, while the second one represents trend strength. The third is a new custom indicator that produces short and long entry signals. It is based on the crossing of two moving averages of different size with a MACD (Moving Average Convergence Divergence) filter. The moving average crossing is expected to detect changes in the current trend of the prices time series, while the MACD is expected to indicate subtle shifts in the strength and direction of a trend. They are described below.

\subsubsection{RSI - Relative Strength Index}

Relative Strength Index (RSI) is a well known technical indicator proposed by Wilder ${ }^{31}$ that represents the momentum of a financial time series. Momentum is the rate of the price increment or falling. Its value ranges between 0 and 100 . RSI at time $t$ is defined as follows: 


$$
R S I=100-\frac{100}{1+R S}
$$

where RS is the relative strength of the last $n$ sessions and it is defined as:

$$
\mathrm{RS}=\frac{\text { Average_profit }_{\mathrm{t}}(\mathrm{n})}{\text { Average_loss }_{\mathrm{t}}(\mathrm{n})}
$$

where Average_profit $(n)$ and Average_loss $_{t}(n)$ are the average of the profits or losses, respectively, obtained in the last $n$ sessions. That is, from time $t$ to time $t-(n-1)$. However, these values are usually estimated using the following smoothing equations:

$$
\begin{aligned}
& \text { Average_profit }_{t}(n)=\frac{\text { Average_profit }_{t-1}(n) \cdot(n-1)+\text { profit }_{t}}{n} \\
& \text { Average_loss }_{t}(n)=\frac{\text { Average_profit }_{t-1}(n) \cdot(n-1)+\text { loss }_{t}}{n}
\end{aligned}
$$

If a session $t$ results in profits then loss $_{t}=0$ and, if it results in loss then profit $t=0$. The RSI compares profits (bearish trending) and losses (bullish trending) of the last $n$ sessions. Wilder recommends a number of sessions $n=14$. A common interpretation of the RSI index is that it suggests oversold state of the asset under analysis for values $<30$ and similarly, overbought states for values $>70$.

\subsubsection{ADX-Average Directional Movement Index}

Average Directional Movement (ADX) measures the strength of a trend and if there is movement in the market ${ }^{31}$. This indicator is calculated as follows:

$$
\mathrm{ADX}=\frac{\mathrm{DI}_{+}-\mathrm{DI}_{-}}{\mathrm{DI}_{+}+\mathrm{DI}_{-}}
$$

The so called Positive Directional Indicator $\left(D I_{+}\right)$and the Negative Directional Indicator $\left(D I_{-}\right)$are given by:

$$
\begin{aligned}
& \mathrm{DI}_{+}=\frac{\mathrm{DM}_{+}}{\mathrm{TR}}, \\
& \mathrm{DI}_{-}=\frac{\mathrm{DM}_{-}}{\mathrm{TR}},
\end{aligned}
$$

where $D M_{+}$and $D M_{-}$are the Plus and Minus Directional Movement, respectively, and $T R$ is the True Range value calculated in the period under consideration. The $D M_{+}$determines the strength of the bullish movement using this rule:

$$
D M_{+}=\left\{\begin{array}{cl}
\text { high }_{t}-\text { high }_{t-1} & \text { if }\left(\text { high }_{t}-\text { high }_{t-1}\right)>\left(\operatorname{low}_{t-1}-\operatorname{low}_{t}\right) \\
0 & \text { if }\left(\text { high }_{t}-\text { high }_{t-1}\right) \leq\left(\operatorname{low}_{t-1}-\operatorname{low}_{t}\right)
\end{array},\right.
$$

where $h i g h_{t}$ and $l o w_{t}$ are the highest and lowest values in session $t$, respectively.

In the same way, $D M_{-}$determines the strength of the bearish movement using this rule: 


$$
D M_{-}=\left\{\begin{array}{cl}
\operatorname{low}_{t-1}-\text { low }_{t} & \text { if }\left(\text { high }_{t}-\text { high }_{t-1}\right)<\left(\text { low }_{t-1}-\text { low }_{t}\right) \\
0 & \text { if }\left(\text { high }_{t}-\text { high }_{t-1}\right) \geq\left(\text { low }_{t-1}-\text { low }_{t}\right)
\end{array},\right.
$$

The definition of the $T R$ is shown in equation (21). It is easy to see that the $T R$ has no effect on ADX (5) as it is present in both the numerator and denominator. However, we have reported the ADX definition as it appears in most trading books.

The ADX ranges between 0 and 100. For values less than 20 it is assumed that the asset analyzed follows a lateral movement. But if ADX is greater than 20, it may be considered that there is a trend in the time series. Values greater than 40 are usually associated with a strong trend. ADX is non-directional so it quantifies the trend strength regardless it is up or down.

\subsubsection{MyMACD - My Moving Average Convergence Divergence}

My Moving Average Convergence Divergence (MyMACD) is a new custom indicator proposed in this work. On the one hand, the MACD indicator is based on the crosses of two exponential moving averages (EMA), one fast (short period) and another one slow (long period). This indicator is expected to detect changes in the trend of the prices ${ }^{32}$ and is usually applied as entry signal for short and long positions. It is a trend-following momentum indicator. We have used the information provided by this technical indicator to create rules that will help to analyze the performance of values confirming a trend. This avoids typical too-sensitive behavior of the moving average crossovers so we can avoid false market entries as far as possible.

The MACD indicator is based on three time series calculated from historical price data. The first time series is the MACD which is estimated at time $t$ as,

$$
\mathrm{MACD}_{\mathrm{t}}=\mathrm{SMA}_{\mathrm{t}}(\mathrm{m}, \text { price_ts })-\mathrm{SMA}_{\mathrm{t}}(\mathrm{n}, \text { price }),
$$

where $m<n$ and $S M A_{t}(p, t s)$ is the Simple Moving Average of $p$ periods estimated at time $t$ for the time series $t s$ :

$$
\mathrm{SMA}=\frac{\mathrm{ts}_{\mathrm{t}}+\mathrm{ts}_{\mathrm{t}-1}+\cdots+\mathrm{ts}_{\mathrm{t}-(\mathrm{p}-1)}}{\mathrm{p}}
$$

In this expression $t s_{t}$ is the current value of the time series $t s$. As it can be seen in Equation (10), the MACD indicator is the difference between two Mas: the slow one, $M A_{t}(m$, price $)$, and the fast one, $M A_{t}(n$, price $)$.

In addition, the MACD indicator uses the Signal time series which is defined at time $t$ as:

$$
\text { Signal }_{t}=\operatorname{EMA}_{t}(s, M A C D) \text {, }
$$


where $E M A_{t}(s, M A C D)$ is the exponential smoothing average of $s$ periods of the MACD. The Exponential Moving Average of $p$ periods at time $t$ for the time series $t s$ is defined as follows:

$$
E M A_{t}(p, t s)=\alpha \cdot t s_{t}+(1-\alpha) E M A_{t-1}(p, t s),
$$

The smoothing factor $\alpha$ is $\alpha=2 /(1+p), t s_{t}$ is the current value of the time series $t s$, and $E M A_{t-1}(p, t s)$ is the previous value of the exponential moving average.

Finally, the MACD indicator uses the divergence time series,

$$
\text { Divergence }_{t}=\text { MACD }_{t}-\operatorname{Signal}_{t}(s, M A C D) \text {. }
$$

The values of $m, n$ and $l$ are usually 12,26 , and 9 days, respectively.

Our MyMACD indicator checks two conditions to determine whether is convenient to enter the market with a long or a short position. If none of them is met then the order "no entry" is suggested. The condition for the long entry is,

$$
\text { Divergence }_{t}>0 \text { \& Divergence } \text { Di-1 } \leq 0
$$

While the condition for the short entry is:

$$
\text { Divergence }_{t}<0 \text { \& Divergence } e_{t-1} \geq 0
$$

If any of the conditions is met, then we estimate the strength of the signal by,

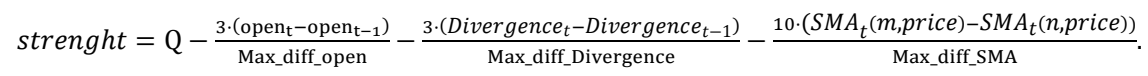

where open $\mathrm{t}_{\mathrm{t}}$ is the open value in session $t$. The reference value $\mathrm{Q}$ is 25 if the condition met is the short one, $\mathrm{Q}=75$ if the long one condition is met, and the strength is 50 if none of the conditions is true.

$$
\begin{gathered}
\text { Max_open_price }=\max _{z} \mid \text { open }_{t}-\text { open }_{t-1} \mid, \forall z=1, \ldots, t, \forall z=1, \ldots, t \\
\text { Max_diff_Divergence }=\max _{z} \mid \text { Divergence }_{t}-\text { Divergence }_{t-1} \mid, \forall z=1, \ldots, t \\
\text { Max_diff_SMA }=\max _{z} \mid \operatorname{SMA}_{t}(m, \text { price })-S M A_{t}(n, \text { price }) \mid, \forall z=1, \ldots, t
\end{gathered}
$$

The output of Equation (17) can be interpreted as follows. The lower the value of Q (less than 25), the stronger the long entry signal. On the contrary, the higher the value (greater than 75 ), the stronger the short entry signal. A value around 50 shows a neutral tendency.

The reason of using SMAs instead of EMAs is the fact that EMAs may provide false entries to the market because of its speed and its ability to represent the most recent price movements. Therefore, SMA is expected to offer a more conservative view than EMAs. 


\subsection{Entry Rules}

The proposed trading system is based on a set of rules that combines the RSI, ADX and MyMACD indicators to generate two output signals, Bullish and Bearish, which are responsible for entering the market. The bullish output indicates that prices are expected to rise high, and therefore, it is suggested to enter the market with a long position. Moreover, the bearish output indicates the prices will drop and then it suggests entering the market with a short position.

In this article we will compare the classic (crisp) version of the trading system with its fuzzy counterpart. The conventional one is based on analytic rules, while the second uses fuzzy rules with the information given by the fuzzification of the indicators. They are described below.

\subsubsection{Rules of the classic trading strategy}

This classical trading system is based on a set of fixed rules. The inputs are positive integer numbers and the outputs are binary values (1: yes, or 0 : not). The inputs express certain combination of conditions that, according to the trader belief, represent a bullish market. In our case the antecedents are the three indicators we are dealing with. Regarding the output, the signal does not advice on the strength level of the entry but only the convenience of entering or exiting the market. The rules are shown in Tables I (bullish) and Table II (bearish):

Table I. Rules of the Classic Trading System: Bullish signal.

\begin{tabular}{lccccccc}
\hline & MyMACD & & RSI & & ADX & & Bullish \\
\hline if & $>15 \&<35$ & $\&$ & $>30 \&<85$ & $\&$ & $>25 \&<55$ & then & 1 \\
elseif & $>15 \&<35$ & $\&$ & $>25 \&<85$ & $\&$ & $>55$ & then & 1 \\
elseif & $>15 \&<35$ & $\&$ & $<25$ & $\&$ & $>25 \&<55$ & then & 1 \\
elseif & $>15 \&<35$ & $\&$ & $<25$ & $\&$ & $<25$ & then & 1 \\
elseif & $>15 \&<35$ & $\&$ & $<25$ & $\&$ & $>55$ & then & 1 \\
elseif & $<15||>85 \|(>35 \&<65)$ & $\&$ & $<25$ & $\&$ & $>25 \&<55$ & then & 1 \\
else & & & & & & then & 0 \\
\hline
\end{tabular}


Table II. Rules of the Classic Trading System: Bearish signal.

\begin{tabular}{lccccccc}
\hline & MyMACD & & RSI & & ADX & & Bearish \\
\hline if & $>65 \&<85$ & $\&$ & $>85$ & $\&$ & $>25 \&<55$ & then & 1 \\
elseif & $>65 \&<85$ & $\&$ & $>85$ & $\&$ & $<55$ & then & 1 \\
elseif & $>65 \&<85$ & $\&$ & $>85$ & $\&$ & $>55$ & then & 1 \\
elseif & $>65 \&<85$ & $\&$ & $>35 \&<85$ & $\&$ & $>25 \&<55$ & then & 1 \\
elseif & $>65 \&<85$ & $\&$ & $>35 \&<85$ & $\&$ & $>55$ & then & 1 \\
elseif & $<15\|>85\|(>35 \&<65)$ & $\&$ & $>85$ & $\&$ & $>25 \&<55$ & then & 1 \\
else & & & & & & then & 0 \\
\hline
\end{tabular}

\subsubsection{Fuzzy trading rules}

Fuzzy logic, by its very nature, tolerates uncertainty in decision making. ${ }^{33}$ In a trading system it can be used to describe the output of technical indicators and trading signals by imprecise linguistic terms. Thus, a trader is able to create a trading system that best represents the expert's belief on market behaviour and that produces a "continuous" output decision using imprecise inputs.

In our system, technical indicators and output conditions have been fuzzified as shown in Figure 1. Three fuzzy sets are defined for the MyMACD input variable: LONG, SHORT and WAIT. They represent long position, short position, and no position at all, respectively. It is important to remark that WAIT, which is between LONG and SHORT, not only represents a market with sideways movement but it is also located at the extremes of the range of possible values of the indicator. This means that very extreme positions are typically followed by a market movement in the opposite direction (Figure 1, top left). Again three fuzzy sets are assigned to the RSI indicator: LOW, MEDIUM and HIGH, depending on the value of this technical indicator (Figure 1, top right). Analogously, the output of the ADX indicator is associated to three fuzzy sets: NOTREND, for low value; ONTREND, for medium values, and DANGER, for high values. The latter represent the current trend is very likely coming to an end (Figure 1, bottom left). The bullish and bearish outputs are described by the fuzzy sets WEAK, STRONG and VERYSTRONG, whose names are selfexplanatory (Figure 1, bottom right). For the sake of simplicity and without losing generality, all the fuzzy sets are represented by triangular membership functions. 


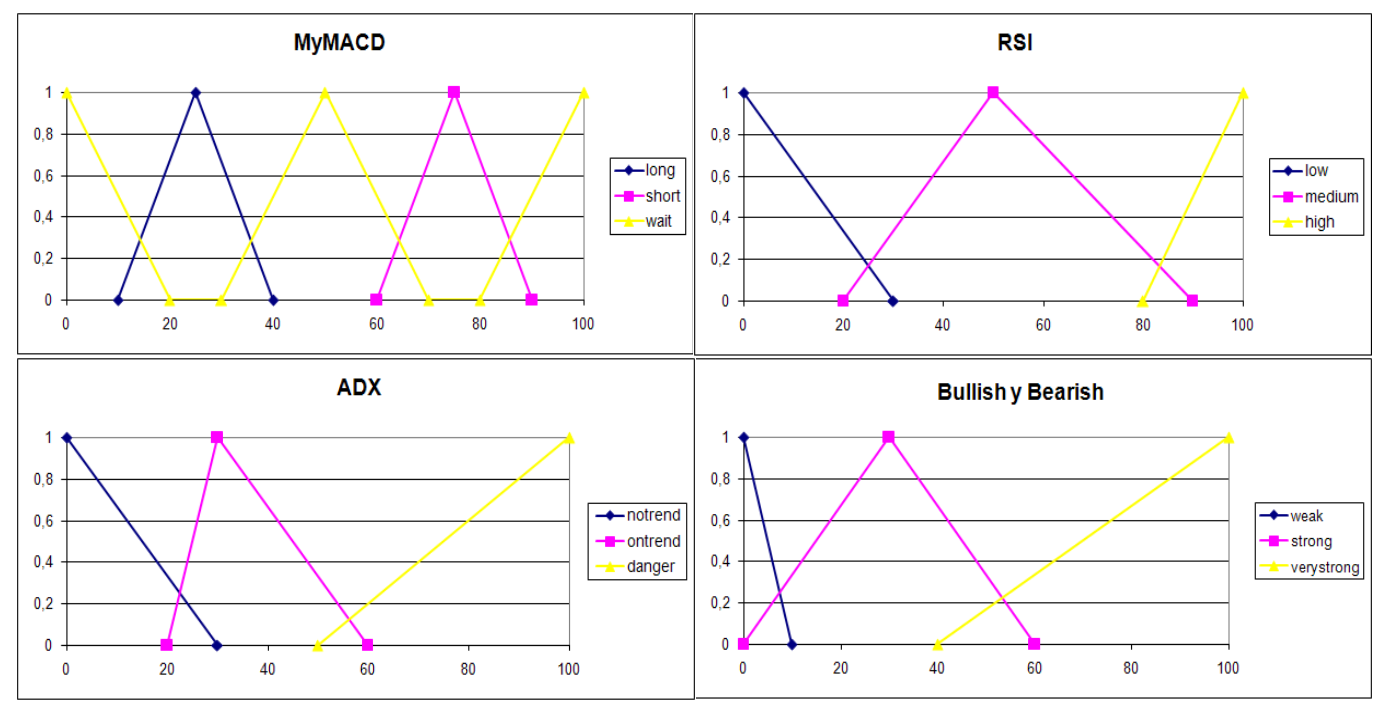

Figure 1. Fuzzification of the indicators and outputs.

Following the experience and expertise of a trader, the following set of if-then rules has been defined (Table III). The rules have two consequents (bullish and bearish), with fuzzy outputs: WEAK, meaning the system should not enter the market; STRONG and VERYSTRONG, which suggest entering the market with different strength level.

Table III. Fuzzy rules of the trading system.

\begin{tabular}{lcccc}
\hline MyMACD & RSI & ADX & Bullish & Bearish \\
\hline LONG & HIGH & ONTREND & WEAK & WEAK \\
LONG & HIGH & NOTREND & WEAK & WEAK \\
LONG & HIGH & DANGER & WEAK & WEAK \\
LONG & MEDIUM & ONTREND & VERYSTRONG & WEAK \\
LONG & MEDIUM & NOTREND & WEAK & WEAK \\
LONG & MEDIUM & DANGER & STRONG & WEAK \\
LONG & LOW & ONTREND & VERYSTRONG & WEAK \\
LONG & LOW & NOTREND & STRONG & WEAK \\
LONG & LOW & DANGER & STRONG & WEAK \\
SHORT & HIGH & ONTREND & WEAK & VERYSTRONG \\
SHORT & HIGH & NOTREND & WEAK & STRONG \\
SHORT & HIGH & DANGER & WEAK & STRONG \\
SHORT & MEDIUM & ONTREND & WEAK & VERYSTRONG \\
SHORT & MEDIUM & NOTREND & WEAK & WEAK \\
SHORT & MEDIUM & DANGER & WEAK & STRONG \\
SHORT & LOW & ONTREND & WEAK & WEAK \\
SHORT & LOW & NOTREND & WEAK & WEAK \\
SHORT & LOW & DANGER & WEAK & WEAK \\
WAIT & HIGH & ONTREND & WEAK & STRONG \\
WAIT & HIGH & NOTREND & WEAK & WEAK \\
WAIT & HIGH & DANGER & WEAK & WEAK \\
WAIT & MEDIUM & ONTREND & WEAK & WEAK \\
WAIT & NEDIUM & NOTREND & WEAK & WEAK \\
WAIT & MEDIUM & DANGER & WEAK & WEAK \\
WAIT & LOW & ONTREND & STRONG & WEAK \\
WAIT & LOW & NOTREND & WEAK & WEAK \\
WAIT & LOW & DANGER & WEAK & WEAK \\
\hline
\end{tabular}


A Mamdani fuzzy system ${ }^{34}$ with operators based on the maximum (or) and minimum (and) is used. The defuzzification is carried out by the centroid method.

\subsection{Exit Conditions}

For both classic and fuzzy trading systems the same exit conditions (stop-loss and stop-profit) are set up. If the system indicates an entry into the market, a protective stop threshold is established. If it is exceeded, market exit takes place. Thus the maximum losses for each trade are limited and will be used for the calculation of capital to invest. Similarly, an income threshold (stop-profit) to determine the market exit is established, but now this exit is due to the fact that it is expected to have exceeded revenues. This will ensure prudential profits avoiding the market taking an unexpected turn.

Both thresholds are estimated according to the price volatility at the moment of the operation. The ATR (Average True Range) of $m$ sessions will be used as volatility indicator. The average of the TR (True Range) is defined as follows:

$$
T R=\operatorname{Max}\left(M_{t}-m_{t}, M_{t}-c_{t-1}, m_{t}-c_{t-1}\right),
$$

where $m$ is obtained by applying an optimization technique, in our case genetic algorithms. The value of $M_{t}$ is the highest price in session $t ; m_{t}$ is the lowest price in session $t$, and $c_{t-1}$ is the close value of the previous session, i.e., $t-1$.

In our system, the stop-loss is the price of market entry minus twice the value of ATR of $m$ sessions. The stop-profit will be the price of market entry plus 1.5 times the ATR of $m$ sessions.

\section{MONEY MANAGEMENT}

So far we have presented the fuzzy trading system with a simple risk management strategy. Now we will use the output of the fuzzy trading rules to make a decision on the money to invest. More precisely, we will use a modification of the optimal-F that uses as input the fuzzy output of the intelligent trading system.

The optimal-F, proposed by Vince, ${ }^{25}$ provides a way to calculate the exact amount to invest in order to produce a geometric growth of profits. It is based on the result of previous trades, increasing the percentage to invest when the winning trades are frequent, and decreasing it when losing trades appear.

The result is a percentage to be applied to the capital available for investment. The optimal-F is calculated finding the fraction $(f)$ that maximizes the Terminal Wealth Relative $(T W R)$ value:

$$
T W R(f)=\prod_{i=1}^{n} H P R_{i}(f)
$$


where $H P R_{i}(f)$ is the holding Period Return, which is the return factor for each operation given by:

$$
H P R_{i}(f)=1+\frac{f \cdot\left(\text { return }_{i}\right)}{\text { Risk_Factor }}
$$

and return $_{i}$ is the result of trade I. Risk_Factor is usually the absolute value of the worst trade result so far.

However, the theoretically optimal value of $f$ is seldom used because it is considered too risky. Therefore, it is the so called liquid-F (or fractional-F) what is applied. This fractional-F is generally $10 \%$ of the optimal-F.

Figure 2 represents the gain curve against the risk assumed. It is possible to observe that the optimalF is close to the highest point of the Gaussian curve. Nevertheless, the liquid-F, $10 \%$ of the later, is located on the left side (less risk, less profit).

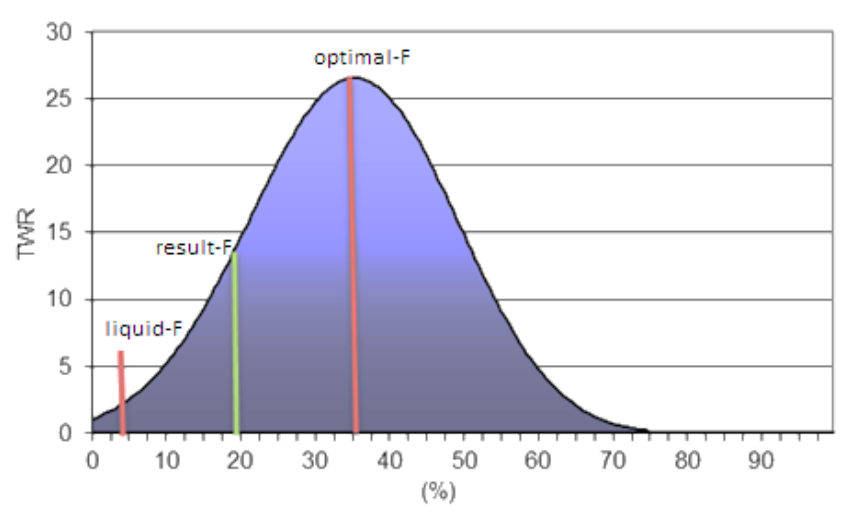

Figure 2. Representation of the optimal-F, result-F and liquid-F.

In our fuzzy trading system, the capital investment will be an amount in that range and it will be estimated as:

$$
\text { custom }_{F}=\text { liquid }_{F}+\frac{(\text { output-threshold }) \cdot \text { range }_{F}}{\text { range }_{\text {ind }}},
$$

where optimal ${ }_{F}$ is the optimal-F value; output is Bullish or Bearish; threshold is the minimum output value of the system that fires the entry (in this case this value is 15); range $_{F}$ is the range between the optimal-F and the liquid-F which is 0.9 optimal $_{F}(90 \%)$, because the liquid-F is 0.1 optimal $_{F}(10 \%)$; range $_{\text {ind }}$ is the range of the system output, which is 85 -the maximum is 100 and the minimum is 15 , as mentioned above-.

Once the optimal-F has been obtained, we have to estimate the number of shares:

$$
\# \text { shares }=\frac{\text { capital } \cdot \text { custom }}{\text { stoploss }} \text {, }
$$


where capital is the available money to be invested and stop - loss is the value used as market exit condition. It is important to take into account that the above formula (25) does consider the share price. In case of not having enough capital to purchase the estimated number of shares, the maximum possible number of shares will be acquired, even if it is less than expected.

\section{EXPERIMENTAL SET UP}

In this section we are going to show and compare the performance of three trading systems. They have been implemented using the Ninja Trader software (NT). The systems are:

- $\quad$ Fuzzy IC: a fuzzy trading where fuzzy information is used for both, the rules and indicators, see Section 2.2.2, and for capital management, see Equations (24) and (25).

- $\quad$ Fuzzy I: an intelligent trading system where fuzzy logic is applied to rules and technical indicators but the capital to invest is estimated using the liquid-F (10\% of the optimal-F).

- Classical: the trading system does not incorporate fuzzy logic neither in the indicators nor in managing the capital. It uses the classic trading rules described in Section 2.2.1 and all the available capital is invested each time.

In addition, the strategy Buy \& Hold $(\mathrm{B} \& \mathrm{H})$ is used as a benchmark, as it is usually done in this field. ${ }^{19,20,27} \mathbf{B} \& \mathbf{H}$ is an investment strategy where traders buy stocks and hold them for a long time. In this case, the entry into the market takes place the first day of the validation period and the marketing exit is the last day.

The trading systems have been tested in two different markets: NASDAQ100 and EUROSTOXX. The validation period goes from the $22^{\text {nd }}$ of December 2012 to the $22^{\text {nd }}$ of December 2013 . These two markets present quite a different behavior during this period. The NASDAQ100 shows a strong bullish behavior in contrast to the EUROSTOXX, which starts moving sideways and then exhibits a bullish behavior. The facto of considering two different markets over a validation period of two years has made possible to test the profitability of our intelligent trading system in different situations.

The first portfolio considers 10 securities of NASDAQ100 market; more precisely, the first 10 securities taken in alphabetical order. These companies are: Apple (AAPL), Adobe (ADBE), ADP (ADP), Autodesk (ADSK), Akamai Technologies (AKAM), Altera (ALTR), Alexion Pharmaceuticals (ALXN), Applied Materials (AMAT), Amgen (AMGN) and Amazon (AMZN). The second portfolio uses a set of 9 securities of EUROSTOXX, chosen from different countries and different sectors: banking, insurance, financial services and real estate. For now, we are not especially interested in obtaining a portfolio with non- 
correlated stocks. The nine companies of EUROSTOXX are: Aegon (AGN), Allianz (ALV), Deutsche Boerse (DB1), Banco de Sabadell (SAB), Santander (SAN), BME (BME), Credit Agricole (ACA), SAMPO (SAMAS) and British Land Company (BLND).

The trading systems will be tested using a sliding window of three months. Each trading system will be trained (optimized) with data from the previous year and it will be validated over a period of three months. After this period, the trading system is again re-optimized. The training and validation periods are shown in Table IV.

Table IV. Training and validation periods using the sliding window.

\begin{tabular}{|c|c|c|c|}
\hline \multicolumn{2}{|c|}{ Training } & \multicolumn{2}{|c|}{ Validation } \\
\hline Start & End & Start & End \\
\hline 22-dec-10 & 22-dec-11 & 22-dec-11 & 22-mar-12 \\
\hline 22-mar-11 & 22-mar-12 & 22-mar-12 & 22-jun-12 \\
\hline 22-jun-11 & 22-jun-12 & 22-jun-12 & 22 -sep-12 \\
\hline 22-sep-11 & 22-sep-12 & 22-sep-12 & $22-$ dec-12 \\
\hline 22-dec-11 & 22 -dec-12 & 22-dec-12 & 22-mar-13 \\
\hline 22-mar-12 & 22-mar-13 & 22-mar-13 & 22-jun-13 \\
\hline 22-jun-12 & 22-jun-13 & 22-jun-13 & 22-sep-13 \\
\hline 22-sep-12 & 22-sep-13 & 22-sep-13 & 22-dec-13 \\
\hline
\end{tabular}

The training period is used to estimate the optimal value of the parameters $m, n$ and $s$, being $m$ and $n$ the number of sessions of the short and long moving average of the MyMACD indicator, respectively; the $s$ value corresponds to the MACD filter. The interval of explored values for each variable is: $m \in[2,25]$, $n \in[15,40]$, and $s \in[1,9]$. The values are calculated using the genetic algorithm included in the NT platform, where the fitness function is the net profit. The configuration parameters of the genetic algorithm are:

- No. of Generations: 5

- Crossover Rate (\%): 80.00

- Generation size: 25

- $\quad$ Mutation Rate (\%): 2.00

- $\quad$ Mutation Strength (\%): 2.00

- $\quad$ Reset Size (\%): 4.00

- $\quad$ Stability Size (\%): 4.00

- $\quad$ Keep best no. results: 10

For the validation period an initial capital of $\$ 6,000$ for each stock is assumed to be available. The net profit during the validation period is shown in Table V (NASDAQ100) and Table VI (EUROSTOXX). The value of each cell means the net profit in dollars (in red meaning a loss). Tables VII, VIII, IX, X, XI and XII show details of the operations for the three approaches (Fuzzy IC, Classical and Fuzzy I) for both, the NASDAQ100 and the EUROSTOXX cases.

In terms of net profit, the Fuzzy IC system is superior to Fuzzy I and Classical. In fact, in the NASDAQ100 market, the classical approach has losses in 5 of the 10 cases; the fuzzy system has losses in 
4 cases, while there are no losses reported in the Fuzzy IC approach. In the EUROSTOXX market, again the net profit is higher for the Fuzzy IC strategy in comparison to the Fuzzy I and Classical approaches. In this case, the Fuzzy I has the minimum number of stocks with losses (two cases in comparison to the three cases obtained with the Fuzzy IC system). Nevertheless, the Fuzzy IC average profit is greater.

Table V. Net profit of the NASDAQ100 stocks.

\begin{tabular}{lccc}
\hline & Classical & Fuzzy I & Fuzzy IC \\
\hline AAPL & $-154,88$ & 340,09 & 556,39 \\
ADBE & $-493,13$ & 613,38 & 1287,51 \\
ADP & 469,53 & 1215,33 & 969,98 \\
ADSK & 315,96 & $-163,54$ & 744,82 \\
AKAM & 877,48 & $-422,76$ & 781,70 \\
ALTR & $-188,11$ & 42,18 & 561,67 \\
ALXN & 96,63 & 467,10 & 368,69 \\
AMAT & $-324,06$ & $-252,83$ & 555,38 \\
AMGN & $-541,27$ & $-368,04$ & 466,41 \\
AMZN & 708,70 & 6,43 & 584,95 \\
\hline
\end{tabular}

Table VI. Net profit of the EUROSTOXX stocks.

\begin{tabular}{lccc}
\hline & Classical & Fuzzy I & Fuzzy IC \\
\hline AGN & 621.90 & 80.91 & -163.58 \\
ALV & 2553.08 & -201.85 & 352.16 \\
DB1 & $-793,79$ & 310.01 & -233.95 \\
SAB & -199.38 & -378.02 & 1044.59 \\
SAN & -926.75 & 755.62 & 792.32 \\
BME & -570.12 & 398.15 & 535.02 \\
ACA & 227.91 & 251.44 & 924.35 \\
SAMAS & 45.66 & 117.05 & 1256.38 \\
BLND & -718.00 & 20.20 & -391.8 \\
\hline
\end{tabular}

The fuzzy approaches perform more trading operations than the classic. It is important to remark that each fuzzy systems has been optimized independently, thus the number of trades are different. In general, the fuzzy IC obtains more positive than negative trades and therefore, the average profit over average loss ratio is usually greater than 1 . This means higher trading operations. These are quite desirable properties for a trading system. However, the maximum drawdown (DD) in each stock is also quite high. The drawdown measures the maximum decrease (in percent) that occurs in the curve of capital during the period considered. Indeed the DD is higher than in its classic counterpart, which might be due to the fact that the classic trading system performs fewer trades. But this disadvantage of the fuzzy IC trading system regarding the DD of particular stocks is not serious, as it can be greatly alleviated if we consider the cumulative performance of stocks set. This might not be the case only if the negative trades tend to occur during the same period. 
Table VII. Trading results for the Fuzzy IC approach (NASDAQ100).

\begin{tabular}{lccccccccc}
\hline & $\begin{array}{c}\text { Net Pro- } \\
\text { fit }\end{array}$ & $\begin{array}{c}\text { Max DD } \\
(\%)\end{array}$ & $\begin{array}{c}\text { Trades } \\
\text { total }\end{array}$ & Trades + & Trades - & $\begin{array}{c}\text { Average } \\
\text { trade }\end{array}$ & $\begin{array}{c}\text { Average } \\
\text { profit } \\
\text { trade }\end{array}$ & $\begin{array}{c}\text { Average } \\
\text { loss trade }\end{array}$ & $\begin{array}{c}\text { Avg } \\
\text { profit/avg } \\
\text { loss }\end{array}$ \\
\hline AAPL & 556.39 & -13.65 & 21 & 11 & 10 & 0.79 & 4.42 & -4.07 & 1.08 \\
ADBE & 1287.51 & -8.73 & 21 & 13 & 8 & 1.58 & 4.07 & -2.65 & 1.54 \\
ADP & 969.58 & -4.58 & 20 & 12 & 8 & 0.79 & 2.37 & -1.61 & 1.47 \\
ADSK & 742.82 & -10.68 & 22 & 15 & 7 & 0.99 & 3.92 & -6.07 & 0.65 \\
AKAM & 781.70 & -22.33 & 14 & 12 & 2 & 1.17 & 4.02 & -11.40 & 0.35 \\
ALTR & 561.67 & -7.44 & 16 & 8 & 8 & 1.14 & 4.05 & -2.87 & 1.41 \\
ALXN & 368.69 & -13.27 & 22 & 12 & 10 & 0.13 & 5.30 & -6.22 & 0.85 \\
AMAT & 555.38 & -12.08 & 24 & 13 & 11 & 0.37 & 3.77 & -3.53 & 1.07 \\
AMGN & 466.41 & -6.43 & 11 & 7 & 4 & 1.37 & 2.86 & -1.88 & 1.52 \\
AMZN & 584.95 & -4.33 & 11 & 7 & 4 & 1.05 & 2.95 & -2.35 & 1.26 \\
\hline
\end{tabular}

Table VIII. Trading results for the Fuzzy I approach (NASDAQ100)

\begin{tabular}{lccccccccc}
\hline & $\begin{array}{c}\text { Net Pro- } \\
\text { fit }\end{array}$ & $\begin{array}{c}\text { Max DD } \\
(\%)\end{array}$ & $\begin{array}{c}\text { Trades } \\
\text { total }\end{array}$ & Trades + & Trades - & $\begin{array}{c}\text { Average } \\
\text { trade }\end{array}$ & $\begin{array}{c}\text { Average } \\
\text { profit } \\
\text { trade }\end{array}$ & $\begin{array}{c}\text { Average } \\
\text { loss trade }\end{array}$ & $\begin{array}{c}\text { Avg } \\
\text { profit/avg } \\
\text { loss }\end{array}$ \\
\hline AAPL & 340.09 & -20.74 & 23 & 13 & 10 & 0.08 & 3.82 & -4.87 & 0.79 \\
ADBE & 613.38 & -10.94 & 24 & 16 & 8 & 0.85 & 3.16 & -4.28 & 0.74 \\
ADP & 1215.33 & -4.58 & 17 & 13 & 4 & 1.41 & 2.18 & -1.20 & 1.82 \\
ADSK & -163.54 & -17.24 & 22 & 10 & 12 & -0.49 & 4.96 & -5.30 & 0.94 \\
AKAM & -422.76 & -15.39 & 20 & 9 & 11 & -1.13 & 3.69 & -4.84 & 0.76 \\
ALTR & 42.18 & -4.65 & 12 & 7 & 5 & 0.09 & 2.24 & -2.69 & 0.83 \\
ALXN & 467.10 & -11.25 & 13 & 9 & 4 & 1.38 & 4.08 & -5.13 & 0.80 \\
AMAT & -252.83 & -9.74 & 24 & 12 & 12 & -0.38 & 3.69 & -3.98 & 0.93 \\
AMGN & -368.04 & -8.18 & 10 & 3 & 7 & -1.43 & 2.50 & -3.48 & 0.72 \\
AMZN & 6.43 & -7.64 & 14 & 8 & 6 & -0.19 & 2.95 & -4.40 & 0.67 \\
\hline
\end{tabular}

Table IX. Trading results for the Classical approach (NASDAQ100).

\begin{tabular}{lccccccccc}
\hline & $\begin{array}{c}\text { Net Pro- } \\
\text { fit }\end{array}$ & $\begin{array}{c}\text { Max DD } \\
(\%)\end{array}$ & $\begin{array}{c}\text { Trades } \\
\text { total }\end{array}$ & Trades + & Trades $-\begin{array}{c}\text { Average } \\
\text { trade }\end{array}$ & $\begin{array}{c}\text { Average } \\
\text { profit } \\
\text { trade }\end{array}$ & $\begin{array}{c}\text { Average } \\
\text { loss } \\
\text { trade }\end{array}$ & $\begin{array}{c}\text { Avg profit / } \\
\text { avg loss }\end{array}$ \\
\hline AAPL & -154.86 & -10.41 & 10 & 5 & 5 & -0.15 & 4.15 & -4.53 & 0.92 \\
ADBE & -493.13 & -10.75 & 9 & 4 & 5 & -0.93 & 3.21 & -4.14 & 0.78 \\
ADP & 469.53 & -3.78 & 13 & 9 & 4 & 0.59 & 2.19 & -2.98 & 0.73 \\
ADSK & 315.96 & -5.30 & 4 & 3 & 1 & 1.32 & 3.52 & -5.30 & 0.66 \\
AKAM & 877.48 & -2.23 & 5 & 3 & 2 & 2.91 & 5.79 & -1.41 & 4.10 \\
ALTR & -188.11 & -5.25 & 5 & 2 & 3 & -0.64 & 4.04 & -3.75 & 1.08 \\
ALXN & 96.63 & -10.55 & 7 & 4 & 3 & 0.40 & 6.24 & -7.76 & 0.80 \\
AMAT & -324.06 & -6.90 & 7 & 3 & 4 & -0.79 & 4.59 & -4.83 & 0.95 \\
AMGN & -541.27 & -5.62 & 8 & 3 & 5 & -1.18 & 2.72 & -3.36 & 0.81 \\
AMZN & 708.70 & -5.88 & 9 & 6 & 3 & 1.33 & 3.94 & -3.79 & 1.04 \\
\hline
\end{tabular}

Table X. Trading results for the Fuzzy IC approach (EUROSTOXX).

\begin{tabular}{lccccccccc}
\hline & $\begin{array}{c}\text { Net } \\
\text { Profit }\end{array}$ & $\begin{array}{c}\text { Max DD } \\
(\%)\end{array}$ & $\begin{array}{c}\text { Trades } \\
\text { total }\end{array}$ & Trades + & Trades - & $\begin{array}{c}\text { Average } \\
\text { trade }\end{array}$ & $\begin{array}{c}\text { Average } \\
\text { profit } \\
\text { trade }\end{array}$ & $\begin{array}{c}\text { Average } \\
\text { loss trade }\end{array}$ & $\begin{array}{c}\text { Avg } \\
\text { profit / } \\
\text { avg loss }\end{array}$ \\
\hline AGN & -163.58 & -14.71 & 25 & 11 & 14 & 3.97 & 3.50 & -3.47 & 1.01 \\
ALV & 352.16 & -5.63 & 20 & 12 & 8 & 1.23 & 3.73 & -2.81 & 1.33
\end{tabular}




\begin{tabular}{lccccccccc} 
DB1 & -233.95 & -20.30 & 18 & 8 & 10 & -0.61 & 3.35 & -3.49 & 0.96 \\
SAB & 1044.59 & -13.64 & 22 & 16 & 6 & 3.46 & 6.52 & -6.48 & 1.01 \\
SAN & 792.32 & -10.75 & 22 & 15 & 7 & 36.76 & 75.09 & -38.95 & 1.93 \\
BME & 535.02 & -10.97 & 20 & 14 & 6 & 1.21 & 3.33 & -3.68 & 0.90 \\
ACA & 924.35 & -6.68 & 16 & 13 & 3 & 4.41 & 6.32 & -4.11 & 1.54 \\
SAMAS & 1256.38 & -7.20 & 22 & 17 & 5 & 1.46 & 3.06 & -3.92 & 0.78 \\
BLND & -391.80 & -4.78 & 17 & 8 & 9 & -0.80 & 2.10 & -2.97 & 0.71 \\
\hline
\end{tabular}

Table XI. Trading results for the Fuzzy I approach (EUROSTOXX).

\begin{tabular}{lccccccccc}
\hline & $\begin{array}{c}\text { Net Pro- } \\
\text { fit }\end{array}$ & $\begin{array}{c}\text { Max DD } \\
(\%)\end{array}$ & $\begin{array}{c}\text { Trades } \\
\text { total }\end{array}$ & Trades + & Trades - & $\begin{array}{c}\text { Average } \\
\text { trade }\end{array}$ & $\begin{array}{c}\text { Average } \\
\text { profit } \\
\text { trade }\end{array}$ & $\begin{array}{c}\text { Average } \\
\text { loss trade }\end{array}$ & $\begin{array}{c}\text { Avg } \\
\text { profit / } \\
\text { avg loss }\end{array}$ \\
\hline AGN & 80.91 & -8.90 & 20 & 10 & 10 & 10.75 & 3.90 & -3.17 & 1.23 \\
ALV & -201.85 & -12.66 & 28 & 14 & 14 & -0.17 & 2.55 & -3.03 & 0.84 \\
DB1 & 310.01 & -6.52 & 14 & 9 & 5 & 0.98 & 3.70 & -4.02 & 0.92 \\
SAB & -378.02 & -20.69 & 23 & 10 & 13 & -0.29 & 6.87 & -6.85 & 1.00 \\
SAN & 755.62 & -10.00 & 18 & 14 & 4 & 38.20 & 61.19 & -22.40 & 2.73 \\
BME & 398.15 & -10.97 & 24 & 16 & 8 & 0.71 & 3.53 & -3.63 & 0.97 \\
ACA & 251.44 & -12.39 & 20 & 12 & 8 & 1.55 & 6.29 & -5.75 & 1.09 \\
SAMAS & 117.05 & -9.05 & 19 & 12 & 7 & -0.08 & 2.19 & -3.61 & 0.61 \\
BLND & 20.20 & -9.96 & 22 & 13 & 9 & 0.05 & 2.76 & -3.72 & 0.74 \\
\hline
\end{tabular}

Table XII. Trading results for the Classical approach (EUROSTOXX).

\begin{tabular}{lccccccccc}
\hline & $\begin{array}{c}\text { Net Pro- } \\
\text { fit }\end{array}$ & $\begin{array}{c}\text { Max DD } \\
(\%)\end{array}$ & $\begin{array}{c}\text { Trades } \\
\text { total }\end{array}$ & Trades + & Trades $-\begin{array}{c}\text { Average } \\
\text { trade }\end{array}$ & $\begin{array}{c}\text { Average } \\
\text { profit } \\
\text { trade }\end{array}$ & $\begin{array}{c}\text { Average } \\
\text { loss trade }\end{array}$ & $\begin{array}{c}\text { Avg } \\
\text { profit } / \\
\text { avg loss }\end{array}$ \\
\hline AGN & 621.90 & -8.26 & 8 & 5 & 3 & 9.79 & 4.10 & -6.38 & 0.64 \\
ALV & 2553.08 & 0 & 9 & 9 & 0 & 4.58 & 4.58 & 0.00 & 0.05 \\
DB1 & -793.79 & -8.71 & 8 & 3 & 5 & -1.67 & 3.22 & -4.74 & 0.68 \\
SAB & -199.38 & -14.96 & 13 & 7 & 6 & -0.23 & 7.87 & -9.16 & 0.86 \\
SAN & -926.75 & -10.47 & 7 & 3 & 4 & -16.35 & 11.25 & -26.44 & 0.43 \\
BME & -570.12 & -8.10 & 12 & 6 & 6 & -0.71 & 3.51 & -4.96 & 0.71 \\
ACA & 227.91 & -18.58 & 10 & 5 & 5 & 0.71 & 9.18 & -8.24 & 1.11 \\
SAMAS & 45.66 & -7.28 & 12 & 9 & 3 & -0.01 & 1.72 & -4.94 & 0.35 \\
BLND & -718.00 & -9.89 & 8 & 3 & 5 & -1.47 & 2.11 & -3.62 & 0.58 \\
\hline
\end{tabular}

As it has been said, we have considered ten NASDAQ100 stocks and nine stocks of EUROSTOXX market, allocating an initial amount of 6,000 monetary units (Euros or dollars, depending on the market) to each stock. The evolution of the capital for the three trading systems in the two portfolios is shown in Figures 3 and 4. The Buy \& Hold strategy has been also represented. It is assumed that shares are bought at the beginning of the validation period and are sold at the end.

Figures 3 and 4 prove that the Fuzzy IC trading system (green line) gives better results than the classical (blue line) and the Fuzzy I (purple line). Nonetheless, although the B\&H strategy (red line) outperforms the other trading systems in terms of capital evolution it presents much higher variability and long drawdown periods, features that are not desirable for a trading system. The B\&H strategy shows that the 
NASDAQ100 portfolio exhibited an upwards trend during the validation period (Figure 3, red line), while for the EUROSTOXX portfolio it presented a sideways performance during the first year and an upwards trend during the second one (Figure 4, red line). The three trading systems implemented obtain profits over the two year period and show a more conservative, i.e., less risky behaviour than the B\&H. Results confirm that the Fuzzy IC trading system is superior to the other two. It obtains higher profits (green line, Figures 3 and 4 ), around $11.45 \%$ for the NASDAQ100 portfolio and $7.62 \%$ for the EUROSTOXX market. It can be considered a very good trading system. On the other hand, the Fuzzy I (purple line) behaves only slightly better than the classic approach (black line), suggesting that a significant part of the improvement is due to the combination of both, the fuzzy indicators and the fuzzy management of the capital.

In addition, it is worth noting that the Fuzzy IC trading system presents a smooth and steady upward trend during the whole validation period. This intelligent decision system also behaves well when the B\&H strategy loses money, that is, when the market goes down. These properties, namely steady profitability and robustness under different circumstances, are quite desirable.

Although the evidence of these experiments may be limited, results are encouraging and suggest that the use of fuzzy logic in trading systems for both the definition of indicators and rules, and capital management should be paid more attention.

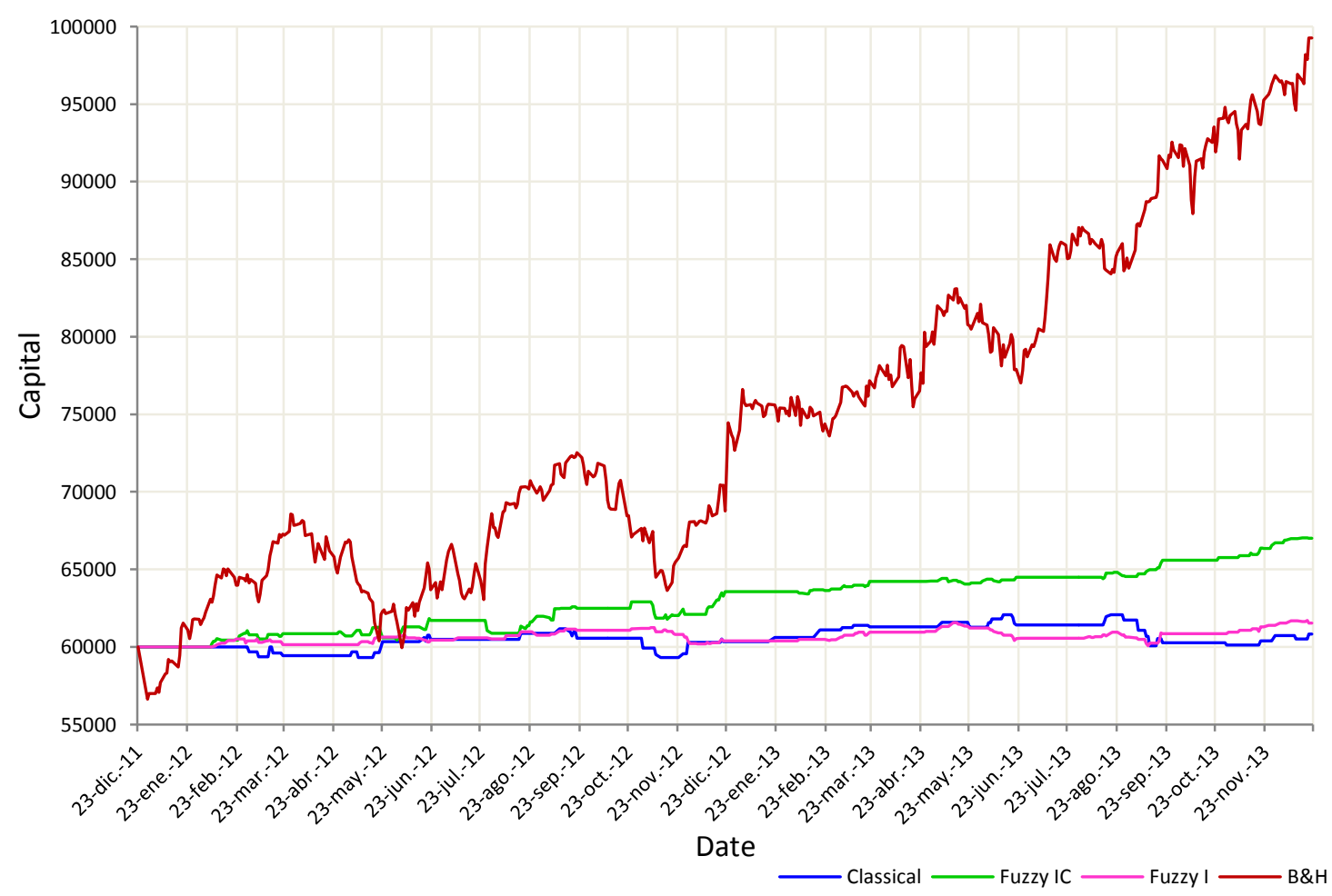

Figure 3. Evolution of the capital for the three trading strategies in the NASDAQ100 portfolio. 


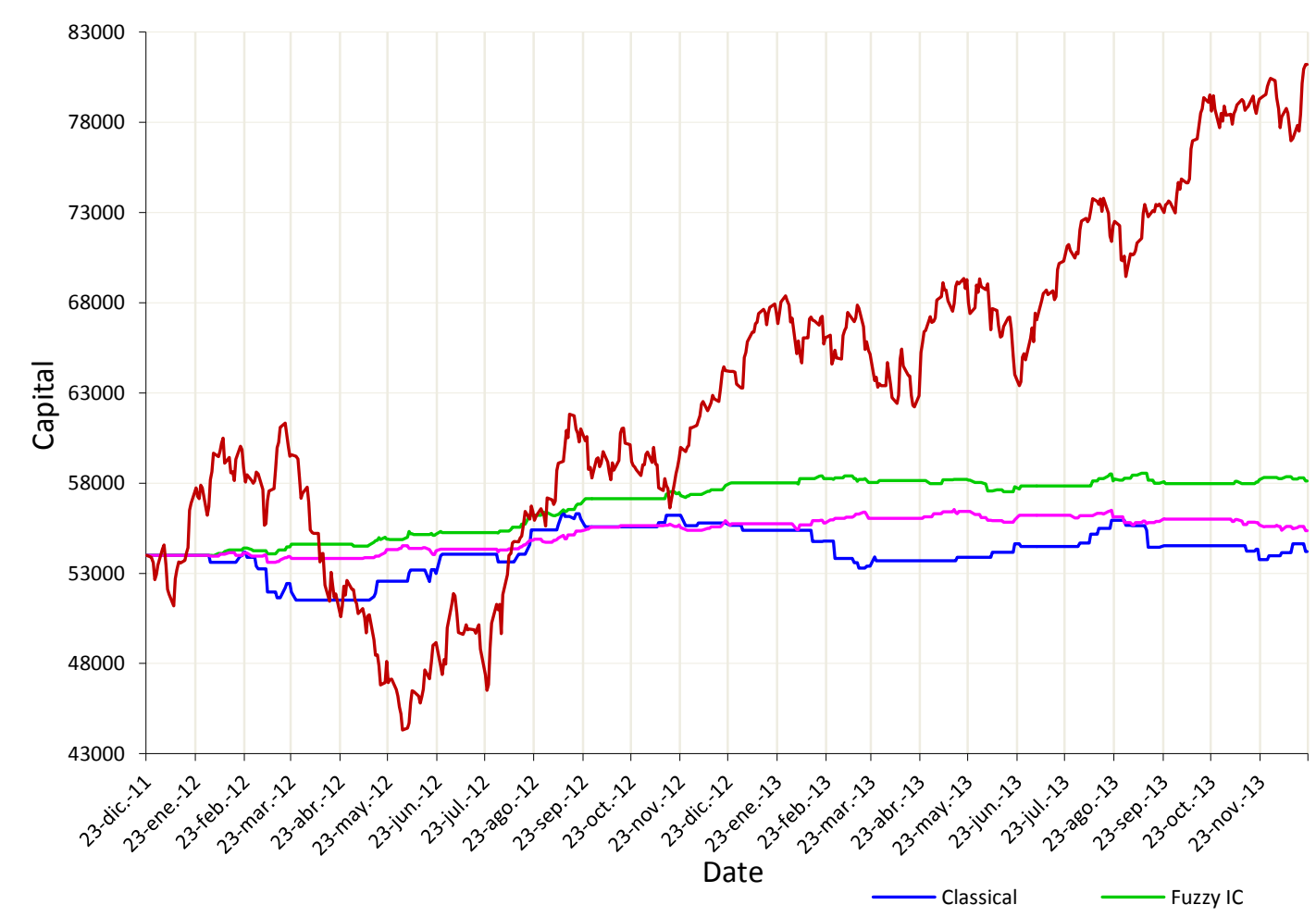

Figure 4. Evolution of the capital for the three trading strategies in the EUROSTOXX portfolio.

\section{CONCLUSIONS}

Because of its ability to deal with uncertainty and vagueness, fuzzy logic is a suitable tool for trading systems. In this paper a trading system with fuzzy indicators (including a new one proposed by the authors), fuzzy trade rules and fuzzy capital management is proposed. Additionally, the trading systems developed in this work not only give advice on when to enter or exit the market, but also on how much to invest. This is remarkable since not many articles in the literature deal with capital management from a fuzzy perspective. The works ${ }^{27,35}$ are interesting and recent exceptions to this fact.

The trading system has been validated over a period of two years in two different portfolios, for different countries and different sectors. Overall, the proposed trading system performs quite well showing a steady upwards trend and obtaining a substantial profit. Evidence suggests that the use of fuzzy capital management is promising and deserves further exploration. In this regard, future work will be to further testing of the system properties of the proposed trading system. The experimental setup will be expanded to include other time periods and more realistic portfolios with uncorrelated assets that belong to different sectors. 
We believe that fuzzy logic is a useful trading tool because it allows the operator to better control decisions on the trading system. In this line, another extension of the present work would be the fuzzification of other classic trading strategies following the ideas in this paper. Thus, it would be possible to validate the appropriateness of the ideas presented here.

Finally, another possible extension of the present work would be the use of fuzzy logic in risk control. For example, to determine the exit conditions with the help of fuzzy logic. This could help to reduce the drawdown observed on the trading of individual assets.

\section{Acknowledgements}

The authors would like to thank Professor Tianrui Li and the anonymous reviewers for their helpful comments. Matilde Santos thanks the Spanish Ministry of Science and Innovation (MICINN) for support under project DPI2013-46665-C2-1-R.

\section{References}

1. Timmermann A, Granger CWJ. Efficient market hypothesis and forecasting. Int J Forecasting 2004; 20:15-27.

2. Brock W, Lakonishok J, LeBaron B. Simple technical trading rules and the stochastic properties of stock returns. J Financ 1992; 47:1731-1764.

3. Gençay R. The predictability of security returns with simple technical trading rules. Journal Empirical Financ 1998; 5(4):347-359.

4. Gençay R. Optimization of technical trading strategies and the profitability in security markets. Econ Lett 1998; 59(2):249-254.

5. Vasileiou E. Is technical analysis profitable even for an amateur investor? Evidence from the Greek stock market (200212). In:Copur Z, editor, Behavioral Finance and Investment Strategies: Decision Making in the Financial Industry. Hershey, PA: IGI Global Publishers; 2014.

6. Sullivan R., Timmermann A, White H. Data-snooping, technical trading rule performance, and the bootstrap. J Financ 1999; 54:1647-1692.

7. Bajgrowicz P, Scaillet O. Technical trading revisited: False discoveries, persistence tests, and transaction costs. J Financ Econ 2012; 106(3):473-491.

8. Allen F, Karjalainen R. Using genetic algorithms to find technical trading rules. J Financ Econ 1999; 51(2):245-271.

9. Bodas-Sagi DJ, Fernández-Blanco P, Hidalgo JI, Soltero-Domingo FJ. A parallel evolutionary algorithm for technical market indicators optimization. Natural computing 2012; 12(2):195-207.

10. White H. Economic prediction using neural networks: the case of IBM daily stock returns. In: Proc. IEEE Int Conf Neural Networks, San Diego, California; 1988:451-458.

11. Vanstone B, Finnie G. Enhancing stock market trading performance with ANNs. Expert Syst Appl 2010; 37(9):66026610. 
12. Lee C-HL, Liu A, Chen WS. Pattern discovery of fuzzy time series for financial prediction. IEEE T Knowl Data En 2006; $18: 613-625$

13. Zhou XS, Dong M. Can fuzzy logic make technical analysis 20/20? Financial Analyst Journal 2004; 60(4):54-75.

14. Atsalakis GS, Valavanis KP. Surveying stock market forecasting techniques-Part II: Soft computing methods. Expert Syst Appl 2009; 36(3):5932-5941.

15. Krollner B, Vanstone B, Finnie G. Financial time series forecasting with machine learning techniques: A survey. In: Eur Symposium on Artif Neural Networks: Computational and Machine Learning, Bruges, Belgium; 2010.

16. Simutis R, Masteika S. Intelligent stock trading systems using fuzzy-neural networks and evolutionary programming methods. Solid State Phenom 2004; 97:59-64.

17. Bagheri A, Peyhani HM, Akbari M. Financial forecasting using ANFIS networks with quantum-behaved Particle Swarm Optimization. Expert Syst Appl 2014; 41(14): 6235-6250.

18. Hadavandi E, Shavandi H, Ghanbari A. Integration of genetic fuzzy systems and artificial neural networks for stock price forecasting. Knowl-Based Syst 2010; 23(8): 800-808.

19. Cheng CH, Chen TL, Wei LY. A hybrid model based on rough sets theory and genetic algorithms for stock price forecasting. Inform Sciences 2010; 180(9):1610-1629.

20. Lam SS, Lam KP, Ng HS. Genetic fuzzy expert trading system for NASDAQ stock market timing. Genetic Algorithms and Genetic Programming in Computational Finance. Springer 2001; 197-217.

21. Kuo RJ, Chen CH., Hwang YC. An intelligent stock trading decision support system through integration of genetic algorithm based fuzzy neural network and artificial neural network. Fuzzy Sets Syst 2001; 118(1):21-45.

22. Chande TS. Beyond technical analysis: How to develop and implement a winning trading system. New York: John Wiley \& Sons; 2001.

23. Kelly JL. A new interpretation of information rate. IRE T Inform Theor; 1956;2(3):185-189.

24. MacLean LC, Thorp EO, Ziemba WT. The Kelly capital growth investment criterion: Theory and practice. World Scientific Handbook in Financial Economic Series vol. 3; Singapore, World Scientific Publishing; 2011.

25. Vince R. Portfolio management formulas. New York: Willey \& Sons; 1990.

26. Jones R. The trading game: playing by the numbers to make millions. New York: Willey \& Sons; 1999.

27. Gradojevic N, Gençay R., Fuzzy logic, trading uncertainty and technical trading. J Bank Financ 2013; 37:578-586.

28. Colby RW. The encyclopedia of technical market indicators ( $2^{\text {nd }}$ ed). McGraw-Hill; 2004.

29. Dourra H, Siy P. Investment using technical analysis and fuzzy logic. Fuzzy Sets Syst 2002; 127(2):221-240.

30. Escobar A, Moreno J, Múnera S. A technical analysis indicator based on fuzzy logic. Electronic Notes in Theor Comput Sci 2013; 292(5):27-37.

31. Wilder JW. New concepts in technical trading systems. Trend Research. Greensboro, N.C.: Hunter Publishing Company 1978.

32. Appel G. Technical analysis power tools for active investors. 2005; NJ, USA: FT Prentice Hall Press.

33. López V, Santos M, Montero J. Fuzzy specification in real estate market decision making. Int J Comp Intelligence Syst $2010 ; 3(1): 8-20$ 
34. Zadeh LA. Fuzzy Sets. Inform Control 1965; 8(3): 338-353.

35. Vella V, Ng WL. A dynamic fuzzy money management approach for controlling the intraday risk-adjusted performance of AI trading algorithms. Intelligent Systems in Accounting, Finance and Management 2014; http://dx.doi.org/10.1002/isaf.1359. 